\title{
Re-engagement in HIV Care: A Clinical and Public Health Priority
}

Richard M Grimes ${ }^{1 *}$, Camden J Hallmark ${ }^{2}$, Kellie L Watkins ${ }^{2}$, Saroochi Agarwal ${ }^{2}$ and Marlene L McNeese ${ }^{2}$

${ }^{1}$ The University of Texas Health Science Center at Houston, Medical School, Houston, USA

${ }^{2}$ The Bureau of HIVISTD and Viral Hepatitis Prevention at the City of Houston Health Department, Houston, USA

\begin{abstract}
As many as $40-50 \%$ of persons living with HIV (PLWH) who once were in HIV care are no longer in care. It is estimated that these individuals account for over $60 \%$ of HIV transmissions. So, preventing the leaving of care and re-engaging PLWH with care are crucial if the HIV epidemic is to be brought under control. Clinicians can improve retention by keeping in close contact with patients. Governmental public health agencies have great expertise in finding and engaging in care persons with sexually transmitted infections. This expertise can be used to re-engage PLWH with HIV care, but it can only be utilized if the agencies know that someone is out of care. Data on who has left care are in the hands of HIV providers. This requires a close working relationship between HIV providers and public health agencies.
\end{abstract}

\section{Introduction}

Public health departments in the United States have been at the forefront of HIV education, testing and surveillance. Effectiveness of education programs is unclear as the reported incidence of HIV has remained relatively constant from 2008 to 2014 at approximately 45,000 $( \pm 3,000)$ cases per year $[1,2]$. The effects of testing and surveillance are much clearer. For example, during 2011 nearly 1.6 million individuals were tested for HIV by public health departments and over 22,000 were found to be HIV positive [3]. In the United States, all HIV infections are reported to local and state health departments where they provide a picture of local HIV trends. These data are then forwarded to the Centers for Disease Control and Prevention and form the basis of an accurate picture of the trends in the epidemic for the whole country [4].

Operating in parallel with public health, HIV clinicians in the United States have coped with a rapidly changing array of medications and guidelines for use in treating persons living with HIV (PLWH). Between 2005 and 2015, the guidelines for treatment of HIV infected adolescents have changed 14 times [5]. Clinicians' use of these medications has changed HIV infection from a death sentence to a chronic disease. In the United States, clinical care of HIV positive persons has been the purview of agencies and/or individuals who were not often affiliated with public health departments; however, clinicians have contributed to surveillance efforts as many providers conduct HIV testing and report cases.

\section{Changing Perspectives}

This separation between clinical medicine and public health has been logical and effective. However, there are three new changes in thinking about the care process of PLWH. The first is based on research that showed that HIV infected persons with low levels of virus are less likely to transmit the virus [6]. This new understanding of transmission dynamics made clinicians essential partners in controlling the epidemic through their use of medications to suppress the virus.

The second shift in thinking was the realization that achieving low viral loads in the community is a process of testing those at risk, linking newly diagnosed HIV positive persons to care, retaining those who entered care, and returning to care those PLWH who have left care [7]. The majority of PLWH are not under the care of HIV providers. Including both those that have never been in care and those that have fallen out of care, it is estimated that about $60 \%$ of PLWH in the United States were not in care for their HIV infection in 2009 At any point in time, one third of those who were once in care are no longer being seen by a HIV provider [8,9]. A study of over 100,000 HIV infected individuals from 13 areas showed that $52 \%$ of people who were in HIV care did not have two visits for HIV care in the previous year although the standard of care was a quarterly visit [10]. A meta-analysis of 28 retention studies showed that $41 \%$ of 75,655 individuals did not have more than $1 \mathrm{HIV}$ care visit over six months [11]. These cross sectional studies did not recognize that the out-of-care population is fluid; a PLWH may enter and leave care multiple times during their infection. Therefore, the numbers of persons who are irregular in their care is likely to be greater than reported in these studies.

The third change in perspective was the recognition that those who have left care are major drivers of the epidemic with one study estimating that PLWH who have left care are responsible for $61.3 \%$ of all HIV transmissions [12]. Individuals who do not take HIV medications and do not suppress their viral load are twenty times more likely to transmit HIV than those with suppressed virus [13]. The majority of PLWH were infected through unprotected sex which may continue for some after their HIV diagnosis. One study showed that $6.3 \%$ of HIV infected males received a syphilis diagnosis within ten years of their HIV diagnosis [14]. A multisite review article showed the average HIV prevalence rate among syphilis patients was $15.7 \%$ [15]. So, some patients are not only having unprotected sex, but they also have open lesions that facilitate onward transmission of the virus. If out-of-care persons are engaging in unprotected sex, they have the elevated viral loads that increase the probability of HIV transmission, and they may have open lesions from a co-occurring sexually transmitted infection (STI) that allows the shedding of HIV during sex.

*Corresponding author: Richard M Grimes, Adjunct professor of Medicine The University of Texas Health Science Center, MSB 1.122, 6431 Fannin Houston, TX 77030, USA, Tel: 77030, 713-661-2007; Fax: 713-500-6722; E-mail: richard.m.grimes@uth.tmc.edu

Received January 19, 2016; Accepted February 10, 2016; Published February 15,2016

Citation: Grimes RM, Hallmark CJ, Watkins KL, Agarwal S, McNeese ML (2016) Re-engagement in HIV Care: A Clinical and Public Health Priority. J AIDS Clin Res 7: 543. doi:10.4172/2155-6113.1000543

Copyright: (c) 2016 Grimes RM, et al. This is an open-access article distributed under the terms of the Creative Commons Attribution License, which permits unrestricted use, distribution, and reproduction in any medium, provided the original author and source are credited. 
This means that finding and returning to care PLWH who have left HIV care is a very high priority for controlling the HIV epidemic.

\section{Implications for Clinicians and Public Health}

Given the likelihood of PLWH leaving care and the high level of risk for transmission by these individuals, there is an obvious need to prevent PLWH from leaving care. There are a number of studies that suggest ways to prevent loss to care. Two review papers examined multiple studies on interventions to improve retention in care $[16,17]$. They described a wide variety of interventions that include personal contact before appointments and when patients miss visits, home visits, education on the importance of staying in care, directly observed therapy, case management, transportation assistance, patient navigators, counseling, mental health services, substance abuse treatment, housing assistance, motivational interviewing and peer mentoring. Most of the studies used more than one method, and it was impossible to determine which part of the intervention was responsible for the outcome. In any event, Okeke et al. reported on nine studies that used a pre-post design and reported improvement in retention in care by PLWH. In seven of the nine studies, the improvement was less than $15 \%$ [16]. These studies provide little guidance for what clinicians may do to retain their patients in care.

However, there are studies that may give guidance on retention. A study evaluated African American and Hispanic youth being cared for at three types of clinics: a usual clinic mixed with patients of all ages, a youth-specific clinic, or a youth-specific clinic enhanced with additional services. This study showed that retention in care as measured by visit consistency nearly doubled with the opening of the youth specific clinic ( $31 \%$ to $57 \%)$. There was a modest improvement when more services were added (65\%), but patients with no gaps in care rose to $96 \%$ with the addition of these services [18]. So, targeting services to a particular group may help in retaining patients. In another study, patients from clinics in six different U.S. cities were randomly assigned to three groups: 1) standard of care, 2) having a staff member assigned to maintain regular contact with the patient (face to face introduction, regular phone calls, appointment reminders and calls after missed visits), or 3) having an assigned contact and receiving a one-hour training in motivational interviewing. Those with an assigned staff person were $10 \%$ more likely to attend at least three consecutive scheduled visits than the standard of care group. The addition of the motivational interviewing component did not improve this. The percent of scheduled visits that were attended was about 5\% higher in the groups with the assigned staff person [19]. A potentially more feasible intervention used computer-generated reminders to clinicians at any time when anomalies occurred in their patients. This included abnormal lab results, detectable viral loads and missed visits. This allowed clinicians to immediately follow up with patients for retesting and rescheduling visits. These patients were compared to those who simply had anomalies placed in their electronic charts. Suboptimal follow up was reduced from $30 \%$ to $20 \%$ by using real time informing of the clinician so that patients could be immediately contacted [20]. What seems to be the most important intervention that prevents leaving care is regular contact with the patient, including prior to appointments and follow up after missed appointments. However, the research seems to show that these interventions only show modest improvements in retention.

So, it would seem that the best efforts of clinicians will not prevent PLWH from leaving care. This has negative health effects on PLWH. HIV infected patients who are not in care or who are in irregular care are far more likely to contract HIV-related opportunistic diseases [21], to develop drug resistant virus [22] and to die [23]. This is a matter of great concern for HIV clinicians; however, they are often overwhelmed with the day-to-day patient care demands and have little time or resources to find those who have left care. Re-engagement in care is also beyond the scope of practice and expertise of clinicians.

Public health departments are the community's experts in locating and linking persons with STIs to care. They have been performing contact tracing to locate sex partners of people with STIs for many years. This expertise could be of great value in controlling HIV transmission if these departments were aware of those PLWH who were no longer in care. Some health departments have used HIV-related laboratory surveillance data to identify individuals with significant gaps between HIV viral loads and/or CD4 counts [24,25]. PLWH who have, for example, greater than six month gaps between these necessary laboratory tests can be identified for follow up. This is feasible for the forty states that require laboratory reporting of CD4 and viral loads [26].

While gaps in lab tests can be a starting point for locating those who have left care, this method has drawbacks. Individuals may have died, become incarcerated or moved out of the area. Laboratories may not be timely in reporting results, leading to misclassifying PLWH who have returned to care before the lab values were reported. Finally, the proper gap to determine when one has left care is not clear. One expert guideline says that laboratory testing be done every "six months for adherent patients who have appropriate values for more than 2-3 years" [27]. Using an interval of six months between laboratory tests may identify adherent PLWH with suppressed virus as having left care. So, conducting follow ups based on laboratory-based surveillance may be futile or inefficient.

Furthermore, using time intervals may not be the best way to measure retention. Mugavero et al. identified six different ways to measure retention in care and applied them to a large cohort of patients from six cities. Three measures used time intervals between visits ranging from four months to a year. These measures correlated poorly with one another. (range $r=0.51$ to $r=0.76$ ). The other measures used missing of scheduled visits as the retention measure. These were highly correlated with one another with $\mathrm{r} \approx 0.85$ [28]. Missing scheduled visits has been shown to predict the risk of mortality [29]. This was true even when using a criterion of time intervals between visits would have classified them as retained in care [30]. So, while public health personnel may be able to use laboratory surveillance data, they may not be using the most clinically relevant information of missed visits, which is only known to providers.

Locating and re-engaging PLWH in care is not easy. Traditional STI contact tracing is based on recent treatment and recent location information. Because there must be a gap of time before a PLWH is defined as not retained in care, individuals are more likely to have relocated. And, unless public health workers are in close contact with HIV providers, the patient location data (address and phone number) in clinic records will not be available. HIV infection also carries with it a significant risk of death, so searches may be a hunt for the deceased. One of the risk factors for HIV transmission is IV drug use which can be associated with criminal behavior, so out-of-care persons may be incarcerated. Also, some PLWH may not wish to be found and will not respond to attempts at contact. Others may be difficult to locate because they are homeless, a condition that is more likely in PLWH $[31,32]$. Evidence of these difficulties is found in the study by Udeagu et al. which concluded that, of 797 PLWH who were identified as having left care because of gaps in their laboratory tests, $14 \%$ were never located. Of those who were located, a third were already back in care, $4 \%$ had moved or were incarcerated, and $2 \%$ had died [33]. 


\section{Conclusion}

While admonitions to practice abstinence and/or safe sex may be helpful, rhetoric is unlikely to significantly alter the spread of HIV. Prevention must focus on those who have left care. Prevention science shows that persons with unsuppressed virus are 20 times more likely to transmit HIV and that out-of-care PLWH are responsible for over $60 \%$ of transmissions [8,13]. So, if every PLWH achieved viral suppression, transmission would be close to nil while the PLWH's health would be greatly improved. Out-of-care persons must be found and re-engaged in care. Unfortunately, this will be unlikely as long as we maintain a system where the relevant data for determining who left care is in the hands of clinicians while the capability for locating and re-engaging individuals in care is with health departments. Clinicians and public health departments have to be partners in preventing HIV transmission.

There are good examples of what can be accomplished when clinicians and public health departments work together. In the state of Washington, there is a program whereby clinicians and the public health department jointly identify and seek PLWH who have left care and re-engage them with care. The program was able to identify those individuals who were in need of re-linkage services by eliminating those who were deceased, had moved out to the area, were incarcerated or had found another provider of HIV care. This allowed providers and health department personnel to concentrate their efforts on those who were actually eligible to be re-engaged in care. When compared to a historical control group, the cooperative program was $70 \%$ more likely to re-engage PLWH with care [34]. Another example of clinical/public health cooperation was conducted in Louisiana. PLWH who had not had a VL or CD4 count in over one year were identified and an alert was placed in the Louisiana State University Health Care Services Division's electronic medical record which notified affiliated clinicians seeing the patient for any disease or condition that the patient may be out of HIV care. Because there was recent location data for these PLWH, contact was efficient and $81.6 \%$ of the PLWH who were in care or who refused to return to care and were, therefore, eligible for re-linkage services were returned to care [35].

Because clinical care is administered by multiple providers in both the public and private sectors, health departments need to take the lead in developing systems that assure PLWH who are out of care reengage with their clinical providers and achieve viral suppression. If public health authorities are not leading in this effort, providers need to contact them and establish ways to involve them in following up on their lost-to-care patients. By working together, clinicians and public health agencies can significantly alter the course of the HIV epidemic.

Funding: This research was supported by a grant from the Merck Foundation and (in part) the Baylor-UTHouston Center for AIDS Research (CFAR), an NIH funded program (AI036211).

\section{References}

1. Centers for Disease Control and Prevention (2012) Diagnoses of HIV Infection in the United States and Dependent Areas.

2. Singh S, Hu X, Wheeler W, Hall HI (2014) HIV diagnoses among men who have sex with men and women-United States and 6 dependent areas, 2008-2011. Am J Public Health 104: 1700-1706.

3. Centers for Disease Control and prevention (2011) HIV testing at CDC funded Sites.

4. Centers for Disease Control and Prevention (2015) Surveillance Overview.

5. AIDS Info. (2015) Archived Adult and Adolescent Guidelines.

6. Granich R, Crowley S, Vitoria M, Smyth C, Kahn JG, et al. (2010) Highly active antiretroviral treatment as prevention of HIV transmission: review of scientific evidence and update. Curr Opin HIV AIDS 5: 298-304.

7. Giordano TP, Suarez-Almazor ME, Grimes RM (2005) The population effectiveness of highly active antiretroviral therapy: are good drugs good enough? Curr HIVIAIDS Rep 2: 177-183.

8. Gardner EM, McLees MP, Steiner JF, Del Rio C, Burman WJ (2011) The spectrum of engagement in HIV care and its relevance to test-and-treat strategies for prevention of HIV infection. Clin Infect Dis 52: 793-800.

\section{AIDS.GOV. HIV (2015) AIDS Care Continuum.}

10. Hall HI, Gray KM, Tang T, Li J, Shouse L, et al. (2012) Retention in care of adults and adolescents living with HIV in 13 U.S. areas. J Acquir Immune Defic Syndr 60: 77-82.

11. Marks G, Gardner LI, Craw J, Crepaz N (2010) Entry and retention in medical care among HIV-diagnosed persons: a meta-analysis. AIDS 24: 2665-2678.

12. Skarbinski J, Rosenberg E, Paz-Bailey G, Hall HI, Rose CE, et al. (2015) Human immunodeficiency virus transmission at each step of the care continuum in the United States. JAMA Intern Med 175: 588-596.

13. Cohen MS, Chen YQ, McCauley M, Gamble T, Hosseinipour MC, et al. (2011) Prevention of HIV-1 infection with early antiretroviral therapy. N Engl J Med 365: 493-505

14. Yang B, Hallmark CJ, Huang JS, Wolverton ML, McNeese-Ward M, et al. (2013) Characteristics and risk of syphilis diagnosis among HIV-infected male cohort a population-based study in Houston, Texas. Sex Transm Dis 40: 957-963.

15. Blocker ME, Levine WC, St Louis ME (2000) HIV prevalence in patients with syphilis, United States. Sex Transm Dis 27: 53-59.

16. Okeke NL, Ostermann J, Thielman NM (2014) Enhancing linkage and retention in HIV care: a review of interventions for highly resourced and resource-poor settings. Curr HIVIAIDS Rep 11: 376-392.

17. Gaston GB, Gutierrez SM, Nisanci A (2015) Interventions that retain African Americans in HIVIAIDS treatment: implications for social work practice and research. Soc Work 60: 35-42.

18. Davila JA, Miertschin N, Sansgiry S, Schwarzwald H, Henley C, et al. (2013) Centralization of HIV services in HIV-positive African-American and Hispanic youth improves retention in care. AIDS Care 25: 202-206.

19. Gardner LI, Giordano TP, Marks G, Wilson TE, Craw JA, et al. (2014) Enhanced Personal Contact With HIV Patients Improves Retention in Primary Care: A Randomized Trial in 6 US HIV Clinics. Clin Infect Dis 59: 725-734.

20. Robbins GK, Lester W, Johnson KL, Chang Y, Estey G, et al. (2012) Efficacy of a clinical decision-support system in an HIV practice: a randomized trial. Ann Intern Med 157: 757-766.

21. Kaplan JE, Hanson D, Dworkin MS, Frederick T, Bertolli J, et al. (2000) Epidemiology of Human Immunodeficiency Virus-Associated Opportunistic Infections in the United States in the Era of Highly Active Antiretroviral Therapy. Clin Infect Dis 30: S5-14.

22. Lucas GM (2005) Antiretroviral adherence, drug resistance, viral fitness and HIV disease progression: a tangled web is woven. J Antimicrob Chemother 55: 413-416.

23. Giordano TP, Gifford AL, White AC Jr, Suarez-Almazor ME, Rabeneck L, et al (2007) Retention in care: a challenge to survival with HIV infection. Clin Infect Dis 44: 1493-1499.

24. Buskin SE, Kent JB, Dombrowski JC, Golden MR (2014) Migration distorts surveillance estimates of engagement in care: results of public health investigations of persons who appear to be out of HIV care. Sex Transm Dis 41: $35-40$.

25. Sweeney P, Gardner LI, Buchacz K, Garland PM, Mugavero MJ, et al. (2013) Shifting the paradigm: using HIV surveillance data as a foundation for improving HIV care and preventing HIV infection. Milbank Q 91: 558-603.

26. Centers for Disease Control and Prevention (2015) State Laboratory Reporting Laws: Viral Load and CD4 Requirements.

27. Aberg JA, Gallant JE, Ghanem KG, Emmanuel P, Zingman BS, et al. (2014) Infectious Diseases Society of America. Primary care guidelines for the management of persons infected with HIV: 2013 update by the HIV medicine association of the Infectious Diseases Society of America. Clin Infect Dis 58: e1-34. 
Citation: Grimes RM, Hallmark CJ, Watkins KL, Agarwal S, McNeese ML (2016) Re-engagement in HIV Care: A Clinical and Public Health Priority. J AIDS Clin Res 7: 543. doi:10.4172/2155-6113.1000543

Page 4 of 4

28. Mugavero MJ, Westfall AO, Zinski A, Davila J, Drainoni ML, et al. (2012) Measuring retention in HIV care: the elusive gold standard. J Acquir Immune Defic Syndr 61: 574-580

29. Horberg MA, Hurley LB, Silverberg MJ, Klein DB, Quesenberry CP, et al. (2013) Missed office visits and risk of mortality among HIV-infected subjects in a large healthcare system in the United States. AIDS Patient Car STDS 27: 442-449.

30. Mugavero MJ, Westfall AO, Cole SR, Geng EH, Crane HM, et al. (2014) Beyond core indicators of retention in HIV care: missed clinic visits are independently associated with all-cause mortality. Clin Infect Dis 59: 1471-1479.

31. Forney JC, Lombardo S, Toro PA (2007) Diagnostic and other correlates of HIV risk behaviors in a probability sample of homeless adults. Psychiatr Serv 58: 92-99.
32. Wenzel SL, Rhoades H, Hsu HT, Golinelli D, Tucker JS, et al. (2012) Behavioral health and social normative influence: correlates of concurrent sexual partnering among heterosexually-active homeless men. AIDS Behav 16: 2042-2050

33. Udeagu CC, Webster TR, Bocour A, Michel P, Shepard CW (2013) Lost or just not following up: public health effort to re-engage HIV-infected persons lost to follow-up into HIV medical care. AIDS 27: 2271-2279.

34. Bove JM, Golden MR, Dhanireddy S, Harrington RD, Dombrowski JC (2015) Outcomes of a Clinic-Based Surveillance-Informed Intervention to Relink Patients to HIV Care J Acquir Immune Defic Syndr 70: 262-268.

35. Magnus M, Herwehe J, Gruber D, Wilbright W, Shepard E, et al. (2012) Improved HIV-related outcomes associated with implementation of a novel public health information exchange. Int J Med Inform 81: e30-38. 